\title{
On initial enhancement of mesospheric dust associated plasma irregularities subsequent to radiowave heating
}

\author{
W. A. Scales and C. Chen \\ The Bradley Department of Electrical and Computer Engineering, Virginia Tech, Blacksburg, VA 24061-0111, USA
}

Received: 1 November 2007 - Revised: 7 May 2008 - Accepted: 7 May 2008 - Published: 5 August 2008

\begin{abstract}
Important observational manifestations of subvisible mesospheric dust are Polar Mesospheric Summer Echoes PMSE which are produced by scattering from electron irregularities produced by dust charging. It has been observed that the PMSE strength can be artificially modified by using a ground-based ionospheric heating facility to perturb the electron irregularity source region that is believed to produce PMSE. Recently it has become evident that significant diagnostic information may be available about the dust layer from the temporal behavior of the electron irregularities during the heating process which modifies the background electron temperature. Particularly interesting and important periods of the temporal behavior are during the turn-on and turnoff of the radiowave heating. Although a number of past theoretical and experimental investigations have considered the turn-off period, the objective here is to consider futher possibilities for diagnostic information available as well as the underlying physical processes. Approximate analytical models are developed and compared to a more accurate full computational model as a reference. Then from the temporal behavior of the electron irregularities during the turn-off of the radiowave heating, the analytical models are used to obtain possible diagnostic information for various charged dust and background plasma quantities.
\end{abstract}

Keywords. Atmospheric composition and structure (Aerosols and particles) - Ionosphere (Ionospheric irregularities; Polar ionosphere) - Space plasma physics (Numerical simulation studies)

\section{Introduction}

Polar Mesospheric Summer Echoes PMSEs are strong radar echoes that have been observed typically in the $50 \mathrm{MHz}$ to

Correspondence to: $\mathrm{W}$. A. Scales

(wscales@vt.edu)
$1.3 \mathrm{GHz}$ frequency range in the summer polar mesosphere (Eklund and Balsley, 1981; Cho et al., 1997). The radar echoes are believed to be produced by Bragg scatter from electron density irregularities at half the radar wavelength. Therefore, the implied wavelengths of the irregularities from past experimental observations are roughly in the range from $10 \mathrm{~m}$ down to $10 \mathrm{~cm}$. The electron irregularities believed to produce PMSE result from electron charging on subvisible dust that exists in the mesosphere. These particles are in the $10 \mathrm{~nm}$ size range and produced from ice since the $85 \mathrm{~km}$ altitude range of PMSE generation is the coldest in the atmosphere. It has been only relatively recently that the existence of these dust particles has been clearly established by in situ space measurements (Havnes et al., 1996). It should be noted that the larger dust particles of $50 \mathrm{~nm}$ size or larger are often observed below the PMSE generation region and make up noctilucent clouds NLCs when observed from the ground and are termed Polar Mesospheric Clouds PMCs when observed from above by spacecraft.

Experiments have shown that PMSE can be perturbed artificially by radiowave heating the source region with a ground-based ionospheric heating facility. The radiowave heating produces an artificial enhancement in electron temperature in the dust cloud region. The first experiments showed a weakening of PMSE upon application of the radiowave heating and recovery afterwards (Chilson et al., 2000; Belova et al., 2001, 2003). It was quickly realized that such modification of PMSE by radiowave heating could be used as a diagnostic for the dust layer parameters. In more recent experiments (Havnes et al., 2003; Havnes, 2004; Biebricher et al., 2006), it was observed that when the artificial electron heating is turned off, the increase in electron density gradients in the dusty plasma will lead to a stronger PMSE, described as "PMSE overshoot". A theoretical model was successfully utilized to predict this behavior and new possibilities for dust layer diagnostics became possible. These works neglected the finite diffusion time of the

Published by Copernicus Publications on behalf of the European Geosciences Union. 
electrons by incorporating a Boltzman model and therefore could not accurately model the temporal evolution immediately after the turn-on of the radiowave heating for a range of observing radar frequencies and dust parameters. A new model was introduced by Chen and Scales (2005) to incorporate finite ambipolar diffusion time effects and address these limitations. The results provided some additional insight into the effects of observing radar frequency, and equivalently irregularity wavelength, on the temporal evolution of the turnoff overshoot. Such variation in the behavior of the irregularities after turn-off was due to the variation in the ambipolar diffusion time relative to the charging time. It was predicted that for sufficiently low observing radar frequencies and appropriate plasma parameters, the prominence of the turn-off overshoot effect may be reduced. Initial possibilities for diagnostic information utilizing such temporal behavior was provided.

It is clear from past work that the temporal evolution of mesospheric dust associated electron irregularities subsequent to radiowave heating has not been exploited to the fullest potential. Possibilities exist for further diagnostic information on the charged dust layer and interpertation of physical processess associated with PMSEs which are considered a forefront issue in upper atmospheric space science. Several past works have provided more qualitative possibilities for diagnostic information, however, the objective of this work is to consider development of approximate analytical models that may allow more quantitiative diagnostic capabilities as well as shed further light on the physical processes associated with the irregularity temporal evolution after the turn-off of radiowave heating. Past computational work will be used as a reference. This work is meant to be a companion to the work of Scales and Chen (2007) which considered the circumstances during the turn-on of radiowave heating. The next section will provide a brief description of past computational work and the basic temporal evolution of the irregularities. Analytical models will then be introduced and discussed which may have possibiities for providing diagnostic information. Finally a summary and conclusion will be provided.

\section{Mesospheric irregularity model}

\subsection{Computational model}

A one-dimensional model is used to study the effects of ionospheric heating by radiowaves on plasma irregularities associated with charged mesospheric dust clouds. The model includes electrons, ions and dust. The dust is taken as immobile and evolution for times much less than the dust diffusion time is considered although the model is not restricted to such time-scales. The plasma model has been described in more detail in previous work (Scales, 2004; Chen and Scales, 2005), therefore only a brief description is provided here.
The ions in the model are described by plasma fluid equations. The ion density is described by the continuity equation

$$
\frac{\partial n_{\mathrm{i}}}{\partial t}+\frac{\partial}{\partial x}\left(n_{\mathrm{i}} v_{\mathrm{i}}\right)=P_{\mathrm{i}}+L_{\mathrm{i}}+\left.\frac{\mathrm{d} n_{\mathrm{i}}}{\mathrm{dt}}\right|_{\text {charging }}
$$

where $x$ is the vertical direction (altitude), and $P_{\mathrm{i}}$ denotes the production of ions by photoionization and $L_{\mathrm{i}}$ denotes the ion loss due to dissociative recombination. This loss term is described by $L_{\mathrm{i}}=\alpha n_{\mathrm{e}} n_{\mathrm{i}}$ where $\alpha$ is the recombination coefficient and $n_{\mathrm{e}}$ is the electron density. The term $\mathrm{dn}_{\mathrm{i}} /\left.\mathrm{dt}\right|_{\text {charging }}$ denotes the ion loss due to the ion flux onto the dust. The electron density is determined from quasineutrality $n_{\mathrm{e}}(x)=n_{\mathrm{i}}(x)-\rho_{\mathrm{d}}(x) / e$ where $\rho_{\mathrm{d}}$ is the dust charge density and $e$ is the unit charge.

Neglecting inertial effects, the ion and electron velocities are given by the momentum equation as

$v_{\alpha}=\frac{1}{v_{\alpha \mathrm{n}}}\left(\frac{q_{\alpha}}{m_{\alpha}} E-\frac{K T_{\alpha}}{m_{\alpha}} \frac{\partial}{\partial x}\left(\log \left(n_{\alpha}\right)\right)\right)$

where $\alpha=i$ or $e$, and $q_{\alpha}, m_{\alpha}$ and $T_{\alpha}$ are the species charge, mass and temperature, and $v_{\alpha \mathrm{n}}$ is the particle-neutral collision frequency. Also, $K$ is Boltzmann's constant. The electrostatic field is denoted by $E$.

The dust is modelled with the standard Particle-In-Cell PIC method (Birdsall and Langdon, 1991) and the dust charge and current densities, $\rho_{\mathrm{d}}$ and $J_{\mathrm{d}}$, respectively, are calculated with the standard PIC techniques. Each dust particle is taken to have a time-varying charge $Q_{\mathrm{d}}(t)$ given by the continuous charging model (Chen and Scales, 2007)

$$
\begin{aligned}
& \frac{d Q_{\mathrm{d}}}{d t}=I_{\mathrm{e}}+I_{\mathrm{i}}, \\
& I_{\mathrm{e}}=\sqrt{8 \pi} r_{\mathrm{d}}^{2} q_{\mathrm{e}} n_{\mathrm{e}} v_{\mathrm{te}} \exp \left(-q_{\mathrm{e}} \phi_{\mathrm{d}} / K T_{\mathrm{e}}\right), \\
& I_{\mathrm{i}}=\sqrt{8 \pi} r_{\mathrm{d}}^{2} q_{\mathrm{i}} n_{\mathrm{i}} v_{\mathrm{ti}}\left(1-q_{\mathrm{i}} \phi_{\mathrm{d}} / K T_{\mathrm{i}}\right),
\end{aligned}
$$

where $r_{\mathrm{d}}$ is the dust radius, $v_{\text {te(i) }}$ is the electron (ion) thermal velocity, and $\phi_{\mathrm{d}}$ is the dust floating potential. The initially uncharged stationary dust is taken to have an irregular density given by

$n_{\mathrm{d}}(x)=n_{\mathrm{d} 0}\left(1+\frac{\delta n_{\mathrm{d} 0}}{n_{\mathrm{d} 0}} \sin (2 \pi m x / \ell)\right)$

where $n_{\mathrm{d} 0}$ is the undisturbed dust density, $\delta n_{\mathrm{d} 0}$ is the dust irregularity amplitude, $m$ is the mode number, and $\ell$ is the system length of the model.

The electrostatic field is calculated from the condition of divergence free currents often used in ionospheric plasmas:

$$
\begin{gathered}
E=\left(\frac{q_{\mathrm{e}} K T_{\mathrm{e}}}{m_{\mathrm{e}} v_{\mathrm{en}}} \frac{\partial n_{\mathrm{e}}}{\partial x}+\frac{q_{\mathrm{i}} K T_{\mathrm{i}}}{m_{\mathrm{i}} \nu_{\mathrm{in}}} \frac{\partial n_{\mathrm{i}}}{\partial x}-J_{\mathrm{d}}\right) \times \\
\left(\frac{q_{\mathrm{e}}^{2}}{m_{\mathrm{e}} v_{\mathrm{en}}} n_{\mathrm{e}}+\frac{q_{\mathrm{i}}^{2}}{m_{\mathrm{i}} \nu_{\mathrm{in}}} n_{\mathrm{i}}\right)^{-1} .
\end{gathered}
$$


The electron irregularities result from electrons attaching to the irregular dust density (Eq. 6). Possibilities of generation of the dust irregularities include neutral air turbulence (Rapp and Lubken, 2003) or plasma instabilities (D'Angelo, 2005). The charge irregularities are ultimately observed as backscattered radar signals and observationally called PMSE under the circumstances described here. For the sinusoidal perturbations used in this work to model the irregularities, the radar reflectivity $\eta(k) \sim \delta n_{\mathrm{e}}^{2}$ where $\delta n_{\mathrm{e}}$ is the electron irregularity amplitude.

Figure 1 shows the temporal evolution of electron irregularities during radiowave heating predicted by the model. Parameters are typical of the mesosphere at $85 \mathrm{~km}$ as described in Chen and Scales (2005) with $n_{\mathrm{e}} \sim 10^{10} \mathrm{~m}^{-3}$ and $T_{\mathrm{e}} \approx 150^{\circ} \mathrm{K}$. The ion mass is taken to be approximately fifty atomic mass units due to the mixture of heavy water cluster ions and $\mathrm{NO}^{+}$at the altitude of interest and $\nu_{\text {in }} \sim 10^{5} \mathrm{~s}^{-1}$ (Lie-Svenson et al., 2003). The dust density is 5\% of the background plasma density and the dust radius is of order $10 \mathrm{~nm}$ to allow an equilibrium dust charge of one electron, $Z_{\mathrm{d} 0}^{e q}=1$, before heating. The irregularity amplitude in the neutral dust density $\delta n_{\mathrm{d} 0} / n_{\mathrm{d} 0}=0.2$. The radiowave heating is "turned on" at $t=25 \mathrm{~s}$ as an instantaneous increase in the electron temperature after an equilibrium electron irregularity amplitude $\delta n_{\mathrm{e} 0}^{e q}$ is reached. The electron temperature is increased by a factor of 5 . The electron temperature is "turned off" at time $t=125 \mathrm{~s}$. The temporal evolution is shown for varying irregularity wavelengths $\lambda$. Assuming an appropriate Bragg scattering wavelength, these correspond to radar frequencies $f=c / 2 \lambda$ where $c$ is the speed of light. It is observed that at shorter irregularity wavelengths (higher radar frequencies) a significant enhancement of the electron irregularity amplitude occurs when the radiowave is turned off at $125 \mathrm{~s}$. The corresponding enhancement in radar echo is known as "PMSE overshoot". The overshoot ultimately results since ambipolar diffusion is more rapid than discharging during the turn-off of the radiowave. This phenomenon has been theoretically predicted as well as experimentally observed and studied in a number of past works (e.g. Havnes et al., 2003; Havnes, 2004; Biebricher et al., 2006). Also, it has been shown that diagnostic information can be obtained from this unique temporal behavior. It can also be seen in Fig. 1 that during the turn-on of the radiowave for longer irregularity wavelengths (lower radar frequencies), an enhancement in irregularity amplitude is predicted (Scales, 2004; Chen and Scales, 2005; Scales and Chen, 2007). This behavior has not been reported in the current literature, however, the prediction of Fig. 1 is that it will require more radar measurements at $\mathrm{HF}$ frequencies during heating experiments to become prominent enough to observe.

The purpose of the present investigation is to consider further diagnostic information that may be obtained from the enhancement of the electron irregularities after the radiowave heating is turned off (i.e. turn-off overshoot). An understanding of the dominant physical processes that drive the

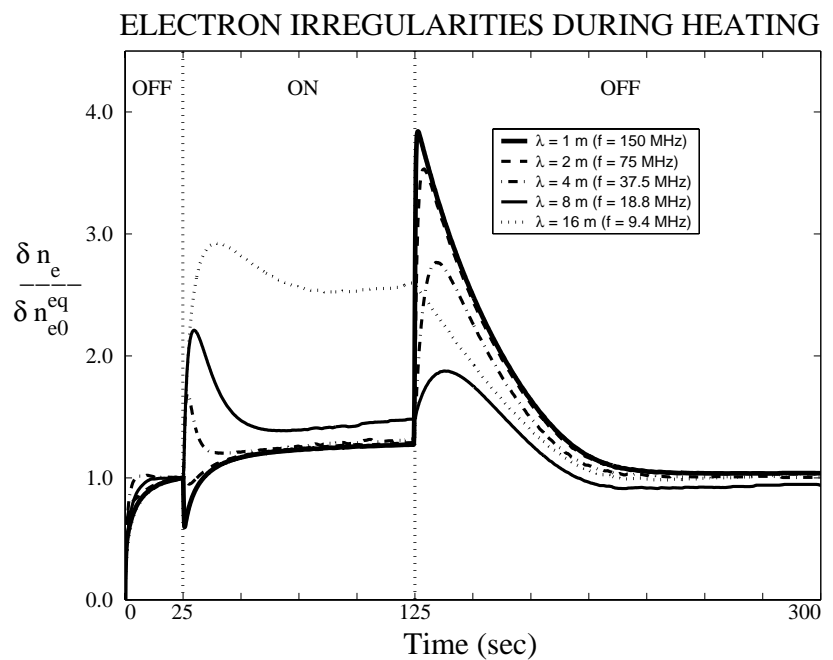

Fig. 1. The time evolution of electron irregularities during radiowave heating with varying irregularity wavelength (and radar frequency) utilizing the computational model. The heating is turned on at $25 \mathrm{~s}$ and off at $125 \mathrm{~s}$.

irregularity temporal evolution during and after the heating process will ultimately allow greater possibilities for diagnostic information. Figure 2 shows the spatial variation of the irregularities in Fig. 1 before radiowave heating (denoted equilibrium), just prior to turn off of the radiowave, and just after turn-off of the radiowave. Three different wavelengths, $\lambda=1,4$ and $16 \mathrm{~m}$, are shown. In each case, the irregularities are shown at $t=24 \mathrm{~s}$ which is the equilibrium state prior to heating. It can be seen that the ion and dust charge irregularities are out of phase with the electron irregularities in each case. For $\lambda=1 \mathrm{~m}$, it can be seen that after the irregularities achieve equilibrium during the heating prior to turn-off at $t=124 \mathrm{~s}$, there is a large enhancement in the ion irregularity amplitude which ultimately results from ambipolar diffusion. The ion irregularity amplitude is significantly larger than the electron irregularity amplitude which also shows some enhancement due to the electron charging flux onto the dust. The dust charge irregularity is in turn enhanced due to the increased charge state of the dust grains from the electron charging. After the radiowave heating at $t=126 \mathrm{~s}$, the electron irregularity amplitude is enhanced (i.e. turn-off overshoot) by approximately a factor of 3 . There is a reduction in the ion irregularity amplitude as well, however, there is little change in the dust charge irregularity amplitude due to the relatively slow discharging due to the small ion flux onto the dust. It is ambipolar diffusion that reduces the large ion irregularity gradients and correspondingly enhances the gradients in the electron irregularities after turn-off of the radiowave. The case for $\lambda=4 \mathrm{~m}$ shows relatively similar behavior to the case with $\lambda=1$. The ambipolar diffusion is slower in this case due to the longer wavelength. It can be seen after the 


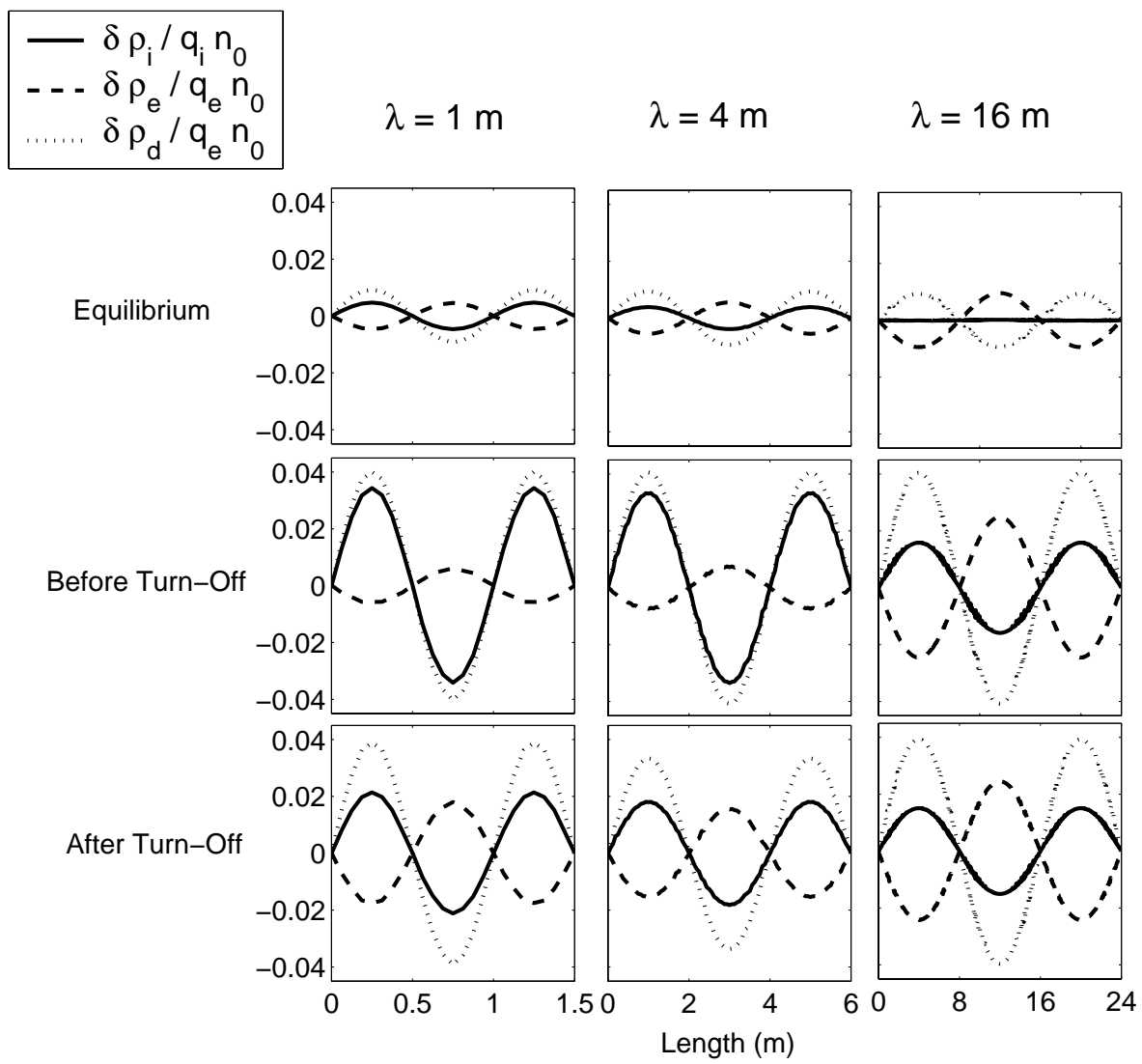

Fig. 2. Spatial presentation of electron $\left(\delta \rho_{\mathrm{e}} / q_{\mathrm{e}} n_{0}\right)$, ion $\left(\delta \rho_{\mathrm{i}} / q_{\mathrm{i}} n_{0}\right)$, and dust $\left(\delta \rho_{\mathrm{d}} / q_{\mathrm{e}} n_{0}\right)$ irregularities before, during, and after radiowave heating with varying irregularity wavelength $(1,4$ and $16 \mathrm{~m})$ as shown in Fig. 1.

turn-off of the heating that the enhancement in the electron irregularities is smaller and roughly a factor of 2 as compared to 3 for $\lambda=1 \mathrm{~m}$. For the longest wavelength case, $\lambda=16 \mathrm{~m}$, there is a much smaller ion irregularity produced both prior and during heating due to the increased ambipolar diffusion time (Lie-Svenson et al., 2003). It is observed that there is little change in the ion irregularity amplitude after turn-off of the radiowave due to reduced ambipolar diffusion effects and consequently no enhancement in the electron irregularities after the turn-off of the radiowave.

The relationship between the electron, ion and dust irregularities can be described from a perturbation analysis (Chen, 1984). For the case of shorter wavelength irregularities (1 and $4 \mathrm{~m}$ ) in Fig. 2, the ambipolar diffusion time is sufficiently small so the electrons and ions may be modeled with the Boltzmann approximation. This implies the normalized electron and ion irregularity amplitudes, $\tilde{\delta} n_{\mathrm{e}}=\delta n_{\mathrm{e}} / n_{\mathrm{e}}$ and $\tilde{\delta} n_{\mathrm{i}}=\delta n_{\mathrm{i}} / n_{\mathrm{i}}$, can be written in terms of the electrostatic potential irregularity amplitude $\delta \phi$ as

$\tilde{\delta} n_{\mathrm{e}} \approx-\frac{T_{\mathrm{i}}}{T_{\mathrm{e}}} \tilde{\delta} n_{\mathrm{i}} \approx \frac{e \delta \phi}{k_{B} T_{\mathrm{e}}}$.
This expression indicates that for sufficiently small irregularity wavelength, the electrons and ions are $180^{\circ}$ out of phase or anti-correlated (Lie-Svenson et al., 2003). Also, during the radiowave heating phase, the ion irregularities are typically larger due to the electron temperature enhancement. The Poisson equation can be used to determine the relationship of the dust irregularity amplitude to the electron irregularity amplitude. In the Fourier spectral domain, the Poisson equation for the irregularities is

$k^{2} \delta \phi \approx \frac{1}{\epsilon_{0}}\left(-e \delta n_{\mathrm{e}}+e \delta n_{\mathrm{i}}-e Z_{\mathrm{d}} \delta n_{\mathrm{d}}\right)$

where the wavenumber is denoted by $k$. Note here that the dust is assumed to be negatively charged with charge number $Z_{\mathrm{d}}$. This implies the relationship

$\tilde{\delta} n_{\mathrm{e}} \approx-\frac{Z_{\mathrm{d}} n_{\mathrm{d}}}{n_{\mathrm{e}}} \frac{1}{1+\lambda_{\mathrm{De}}^{2} / \lambda_{\mathrm{Di}}^{2}+\lambda_{\mathrm{De}}^{2} k^{2}} \tilde{\delta} n_{\mathrm{d}}$

where $\lambda_{\text {De, }}$ is the electron (ion) Debye length. Therefore the electron irregularities are also $180^{\circ}$ out of phase with dust irregularities (which are in phase with the ion irregularities).

The previous results illuminate the relative importance of ambipolar diffusion and charging times in the temporal 
evolution of electron irregularities subsequent to radiowave heating. Utilizing these concepts from the previous computational results, a simplified analytical model will now be developed to consider possible diagnostic information during the turn-off of radiowave heating.

\subsection{Analytical model for irregularity temporal evolution}

The electron density is determined from the ion and dust charge densities using quasi-neutrality. After turning off the radiowave heating, the dominant physical effects are ion density gradient driven ambipolar diffusion and discharging of the dust grains due to ion currents as described in Fig. 2. Therefore, an analytical model for the electron density after turn-off of the radiowave heating can be written in the form (Scales and Chen, 2007)

$$
\begin{aligned}
n_{\mathrm{e}}(t)= & n_{\mathrm{i}}(t)-Z_{\mathrm{d}}(t) n_{\mathrm{d}} \\
= & \left(n_{\mathrm{i} 0}-n_{\mathrm{i} 0}^{e q}\right) e^{-k_{\mathrm{i}} n_{\mathrm{d} 0} t} e^{-t / \tau_{\mathrm{d}}}+n_{\mathrm{i} 0}^{e q} \\
& -\left[\left(Z_{\mathrm{d} 0}-Z_{\mathrm{d} 0}^{e q}\right) e^{-k_{\mathrm{i}} n_{\mathrm{d} 0} t}+Z_{\mathrm{d} 0}^{e q}\right] n_{\mathrm{d} 0}
\end{aligned}
$$

where now the turn-off of the radiowave is referenced to time $t=0$. Here $\tau_{\mathrm{d}} \approx(\lambda / 2 \pi)^{2} / D_{\mathrm{a}}$ is the diffusion time which depends on the irregularity wavelength $\lambda$ and $D_{\mathrm{a}} \approx\left(K T_{\mathrm{i}} / m_{\mathrm{i}} v_{\mathrm{in}}\right)\left(1+T_{\mathrm{e}} / T_{\mathrm{i}}\right)\left(1+Z_{\mathrm{d} 0} n_{\mathrm{d} 0} / n_{\mathrm{e} 0}\right)$ which is the ambipolar diffusion coefficient. Since the dust is essentially motionless on these timescales, the ambipolar diffusion coeficient is appropriate for considering the diffusion processes. After the turn-off of the radiowave, the ion current onto the dust grains dominates the electron current and results in discharging of the dust grains as seen in Fig. 2. The rate coefficient for attachment of ions onto the dust at the initial turn-off of the radiowave is approximated with the ion charging current in Eq. (5) as $k_{\mathrm{i}} \approx I_{\mathrm{i}} /\left(e n_{\mathrm{i}}\right)=\sqrt{8 \pi} r_{\mathrm{d}}^{2} v_{\text {ti } 0}(1-x)$ where $x$ is determined from the equilibrium $I_{\mathrm{e}}+I_{\mathrm{i}}=0$ just prior to turnoff of the radiowave. It should be noted that $x$ depends on the ratio of the electron temperature during and before the radiowave heating is turned on (Chen and Scales, 2005) denoted by $T_{\mathrm{e}} / T_{\mathrm{e} 0}$. It is assumed in the above model that the electron irregularities have achieved steady state prior to the radiowave heating being turned off. Therefore in Eq. (11), $n_{\mathrm{i} 0}$ and $Z_{\mathrm{d} 0}$ are the steady state ion density and charge on the dust grains just prior to turning off the radiowave. The equilibrium values of the ion density and dust grain charge before heating are denoted by $n_{\mathrm{i} 0}^{e q}$ and $Z_{\mathrm{d} 0}^{e q}$, respectively. Recall that the model assumes a dust density that is constant in time $n_{\mathrm{d} 0}$ as described in the previous section. The steady state ion density prior to turning off the radiowave can be written as $n_{\mathrm{i} 0}=n_{\mathrm{e} 0}+Z_{\mathrm{d} 0} n_{\mathrm{d} 0}$ while the equilibrium value before radiowave heating can be written as $n_{\mathrm{i} 0}^{e q}=n_{\mathrm{e} 0}^{e q}+Z_{\mathrm{d} 0}^{e q} n_{\mathrm{d} 0}$ assuming negatively charged dust. Therefore, the temporal evolution of the electron density can be rewritten as

$$
\begin{aligned}
n_{\mathrm{e}}(t)= & e^{-k_{\mathrm{i}} n_{\mathrm{d} 0} t}\left[\left(n_{\mathrm{e} 0}-n_{\mathrm{e} 0}^{e q}\right) e^{-t / \tau_{\mathrm{d}}}\right. \\
& \left.-\left(Z_{\mathrm{d} 0}-Z_{\mathrm{d} 0}^{e q}\right) n_{\mathrm{d} 0}\left(1-e^{-t / \tau_{\mathrm{d}}}\right)\right]+n_{\mathrm{e} 0}^{e q} .
\end{aligned}
$$

Using Eq. (12) and noting the irregularity phases of Fig. 2, the electron irregularity temporal evolution can then be written as

$$
\begin{aligned}
\delta n_{\mathrm{e}}(t) & =e^{-k_{\mathrm{i}} n_{\mathrm{d} 0}}\left[\left(n_{\mathrm{e} 0}+\delta n_{\mathrm{e} 0}-n_{\mathrm{e} 0}^{e q}-\delta n_{\mathrm{e} 0}^{e q}\right) e^{-t / \tau_{\mathrm{d}}}\right. \\
& \left.-\left(Z_{\mathrm{d} 0}-Z_{\mathrm{d} 0}^{e q}\right)\left(n_{\mathrm{d} 0}-\delta n_{\mathrm{d} 0}^{e q}\right)\left(1-e^{-t / \tau_{\mathrm{d}}}\right)\right] \\
& +n_{\mathrm{e} 0}^{e q}+\delta n_{\mathrm{e} 0}^{e q} \\
& -e^{-k_{\mathrm{i}} n_{\mathrm{d} 0} t}\left[\left(n_{\mathrm{e} 0}-n_{\mathrm{e} 0}^{e q}\right) e^{-t / \tau_{\mathrm{d}}}\right. \\
& \left.-\left(Z_{\mathrm{d} 0}-Z_{\mathrm{d} 0}^{e q}\right) n_{\mathrm{d} 0}\left(1-e^{-t / \tau_{\mathrm{d}}}\right)\right] \\
& -n_{\mathrm{e} 0}^{e q}
\end{aligned}
$$

where the electron and dust densities have been written of the form $n_{0}+\delta n_{0}$ which represents the background and perturbation components, respectively. The preceding form can be simplified to

$$
\Delta \tilde{\delta} n_{\mathrm{e}}(t)=e^{-t / \tau_{\mathrm{r}}}\left[e^{-t / \tau_{\mathrm{d}}}+\frac{\Delta Z_{\mathrm{d} 0} \delta n_{\mathrm{d} 0}}{\Delta \delta n_{\mathrm{e} 0}}\left(1-e^{-t / \tau_{\mathrm{d}}}\right)\right]
$$

where in $\Delta \delta n_{\mathrm{e}}(t)=\delta n_{\mathrm{e}}(t)-\delta n_{\mathrm{e} 0}^{e q}$ is the difference in the electron irregularity amplitude from the equilibrium value and $\Delta \tilde{\delta} n_{\mathrm{e}}(t)=\Delta \delta n_{\mathrm{e}}(t) / \Delta \delta n_{\mathrm{e} 0}$ is its value normalized to 1 at $t=0$ where $\Delta \delta n_{\mathrm{e} 0}=\delta n_{\mathrm{e} 0}-\delta n_{\mathrm{e} 0}^{e q}$. Also, $\Delta Z_{\mathrm{d} 0}=Z_{\mathrm{d} 0}-Z_{\mathrm{d} 0}^{e q}$ is the net gain in electron charges during radiowave heating. The reduction time of the ion density due to the dust charging from ion flux is denoted by $\tau_{\mathrm{r}}=1 / k_{\mathrm{i}} n_{\mathrm{d} 0}$. It is noted that $\tau_{\mathrm{r}}$ is related to the dust (dis)charging time after the radiowave heating is turned off by $\tau_{\mathrm{r}}=\tau_{\mathrm{c}}\left(n_{\mathrm{i}} / n_{\mathrm{d}}\right)$.

The approximate analytical model in Eq. (14) can be used to investigate the temporal behavior of the dust associated electron irregularities subsequent to radiowave heating. The condition for an enhancement of the electron irregularities after turn-off of the radiowave (i.e. "turn-off overshoot", (Havnes et al., 2003; Havnes, 2004)) can be obtained from the condition $\partial\left(\Delta \tilde{\delta} n_{\mathrm{e}}\right) / \partial t>0$ for $t=0$ which yields the condition

$$
\frac{\tau_{\mathrm{r}}}{\tau_{\mathrm{d}}}>\frac{1}{\Delta Z_{\mathrm{d} 0} \delta n_{\mathrm{d} 0} / \Delta \delta n_{\mathrm{e} 0}-1} .
$$

Note that this quantitative condition provides a lower diagnostic bound on the ratio of the ion density reduction to ambipolar diffusion time ratio and is a refined quantitative version of the more qualitative condition discussed in previous work for the existence of the turn-off overshoot (Chen and Scales, 2005). This bound can be used to provide diagnostic information on such quantities as dust density, dust radius, dust charge, and electron temperature increase, through the functional dependences of $\tau_{\mathrm{d}}$ and $\tau_{\mathrm{r}}$ on these parameters as described in previous work. 


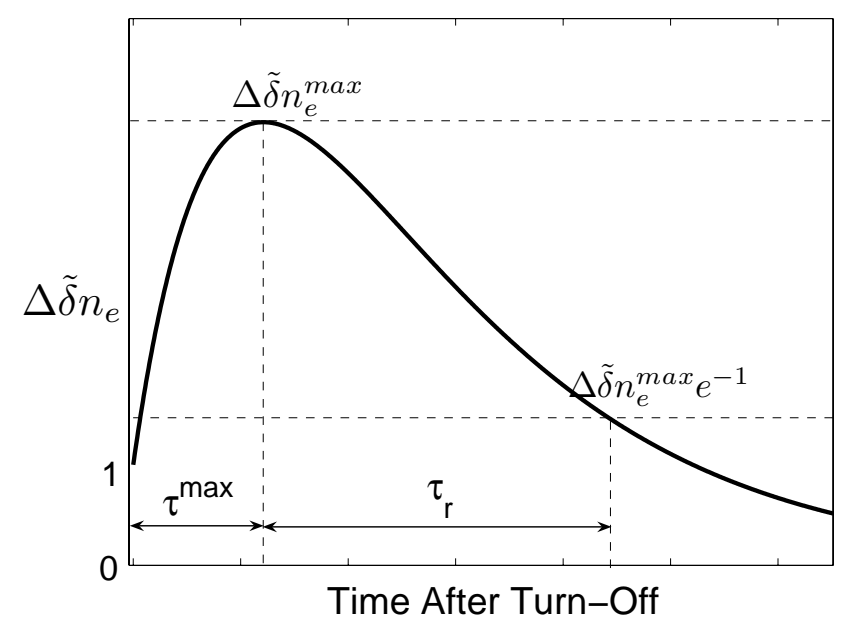

Fig. 3. Schematic of electron irregularity behavior subsequent to radiowave heating.

The time at which the maximum amplitude is reached after the turn-off of the radiowave can be obtained from the condition $\partial\left(\Delta \widetilde{\delta} n_{\mathrm{e}}\right) / \partial t=0$ as

$\tau^{\max } \approx \tau_{\mathrm{d}} \log \left[\left(1+\frac{\tau_{\mathrm{r}}}{\tau_{\mathrm{d}}}\right)\left(1-\frac{\Delta \delta n_{\mathrm{e} 0}}{\Delta Z_{\mathrm{d} 0} \delta n_{\mathrm{d} 0}}\right)\right]$.

This expression can be used to approximate the maximum amplitude of the electron irregularities after the turn-off of the radiowave heating. Substituting Eq. (16) into (14) implies

$$
\begin{aligned}
\Delta \tilde{\delta} n_{\mathrm{e}}^{\max } \approx & \frac{\Delta Z_{\mathrm{d} 0} \delta n_{\mathrm{d} 0}}{\Delta \delta n_{\mathrm{e} 0}}\left[1+\frac{\tau_{\mathrm{d}}}{\tau_{\mathrm{r}}}\right]^{-1} \times \\
& {\left[\left(1+\frac{\tau_{\mathrm{r}}}{\tau_{\mathrm{d}}}\right)\left(1-\frac{\Delta \delta n_{\mathrm{e} 0}}{\Delta Z_{\mathrm{d} 0} \delta n_{\mathrm{d} 0}}\right)\right]^{-\tau_{\mathrm{d}} / \tau_{\mathrm{r}}} . }
\end{aligned}
$$

Assuming before heating, $T_{\mathrm{e}} \approx T_{\mathrm{i}}$, the Boltzmann approximation holds, and $k^{2} \lambda_{\mathrm{De}}^{2} \ll 1$, then Eq. (10) leads to the useful approximation

$$
\frac{Z_{\mathrm{d} 0} \delta n_{\mathrm{d} 0}}{\delta n_{\mathrm{e} 0}} \approx 1+\frac{T_{\mathrm{e}}}{T_{\mathrm{e} 0}}
$$

Using the approximation in Eq. (18), the maximum amplitude after turn-off of the radiowave can be written in terms of the temperature enhancement $T_{\mathrm{e}} / T_{\mathrm{e} 0}$ during heating as

$$
\begin{aligned}
\Delta \tilde{\delta} n_{\mathrm{e}}^{\max } \approx & {\left[\frac{T_{\mathrm{e}} / T_{\mathrm{e} 0}+1-2 \tilde{\delta} n_{\mathrm{e} 0}^{e q}}{\left(1-\tilde{\delta} n_{\mathrm{e} 0}^{e q}\right)\left(1+\tau_{\mathrm{d}} / \tau_{\mathrm{r}}\right)}\right] \times } \\
& {\left[\frac{\left(1+\tau_{\mathrm{r}} / \tau_{\mathrm{d}}\right)\left(T_{\mathrm{e}} / T_{\mathrm{e} 0}-\tilde{\delta} n_{\mathrm{e} 0}^{e q}\right)}{T_{\mathrm{e}} / T_{\mathrm{e} 0}+1-2 \tilde{\delta} n_{\mathrm{e} 0}^{e q}}\right]^{-\tau_{\mathrm{d}} / \tau_{\mathrm{r}}} }
\end{aligned}
$$

where $\tilde{\delta} n_{\mathrm{e} 0}^{e q}=\delta n_{\mathrm{e} 0}^{e q} / \delta n_{\mathrm{e} 0}$. After the maximum value has been achieved (14), it can be seen that there is a decay in the amplitude of the electron irregularities. The approximate temporal behavior after the maximum is reached is given by

$$
\Delta \tilde{\delta} n_{\mathrm{e}}(t) \approx \Delta \tilde{\delta} n_{\mathrm{e}}^{\max } e^{-t / \tau_{\mathrm{r}}} .
$$

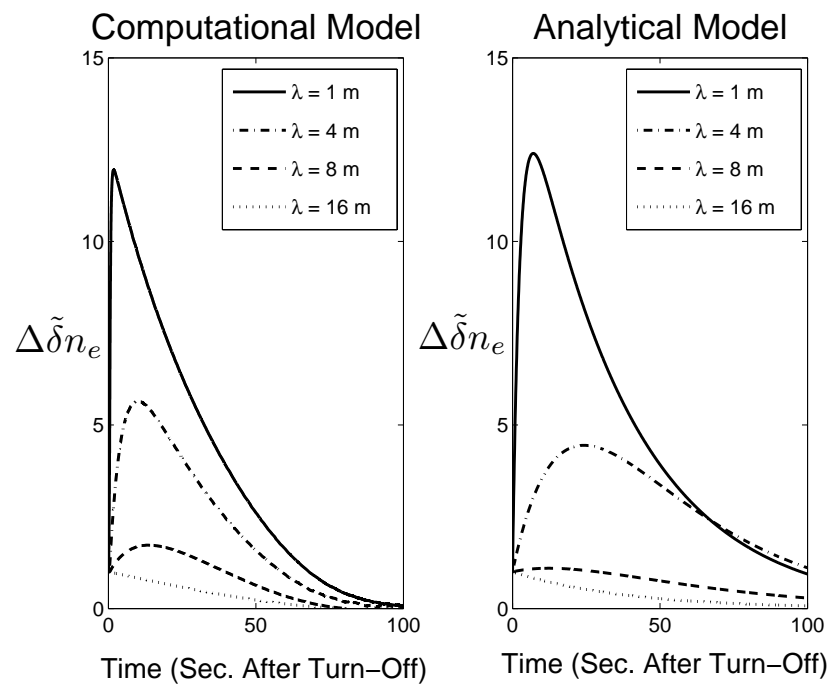

Fig. 4. Comparison of the full computational model of Sect. 2.1 with the analytical model (Eq. 14) for the parameters of Fig. 1. Note results represented in the form of $\Delta \tilde{\delta} n_{\mathrm{e}}$ which is the normalized electron irregularity amplitude difference from the equilibrium before heating.

Therefore the timescale for the decay of the irregularities, after the maximum is reached, is the ion density reduction time $\tau_{\mathrm{r}}$. Therefore $\tau_{\mathrm{r}}$ can also be interpreted as being related to the dust grain discharging time due to ion currents.

From the previous analysis, it can be seen that at least three characteristics of the irregularity temporal evolution after turn-off may be used for diagnostic information. They are the time at which maximum amplitude is reached, Eq. (16), the maximum amplitude achieved, Eqs. (17) and (19), and the timescale for decay after the maximum amplitude has been reached, Eq. (20). It should be noted that Eq. (16) may also be written in terms of the temperature enhancement $T_{\mathrm{e}} / T_{\mathrm{e} 0}$ using Eq. (18). Figure 3 shows a schematic of these characteristics after turn-off.

Figure 4 shows a comparison between the computational model of Sect. 2.1 and the simplified analytical model (Eq. 14). The same cases shown in Fig. 1 are presented, however, the results are expressed in terms of $\Delta \tilde{\delta} n_{\mathrm{e}}$. It is observed that there is quite reasonable agreement between the two models considering the simplifying assumptions used and the approximations on the diffusion and charging timescales which in reality vary in time. There is a tendency for the analytical model to slightly overestimate $\tau^{\max }$ in this regard. The suppression of the turn-off overshoot effect for $\lambda=16 \mathrm{~m}$ is also well predicted in the analytical model from Eq. (15). From these results, confidence can be given for the simplified analytical model providing reasonable accuracy for diagnostic information. 


\section{Conclusions}

It is clear that active perturbation of the source region of electron irregularities that produce PMSEs will provide a rich source of diagnostic information about the corresponding mesospheric dust layer as well as insight on the physical processes that produce PMSEs. This work has attempted to provide further physical insight into the physical processes associated with temporal evolution of the electron irregularities during the turn-off of the radiowave heating. It can be seen to be complementary to past work that has considered the physical processes during turn-on of radiowave heating (Scales and Chen, 2007). The simplified analytical models here provide quite reasonable agreement with the full computational model results and allow information about the dust layer to be readily obtained from the temporal evolution after the turn-off of the radiowave. At least three observable parameters, $\tau^{\max }, \Delta \tilde{\delta} n_{\mathrm{e}}^{\max }$, and $\tau_{\mathrm{r}}$ may provide information on the dust layer such as dust density, dust radius, and charge state. Also possibilities may exist for information on the degree of the temperature enhancement. The results also indicate that future planned experiments involving multiple observing radar frequencies may also contribute to further diagnostic capabilities.

Acknowledgements. This work was supported by the National Science Foundation through grant ATM-0641200.

Topical Editor K. Kauristie thanks two anonymous referees for their help in evaluating this paper.

\section{References}

Belova, E., Chilson, P. B., Rapp, M., and Kirkwood, S.: Electron temperature dependence of PMSE power, Adv. Space Res., 28, 1077-1082, 2001.

Belova, E., Chilson, P. B., Rapp, M., and Rietveld, M. T.: The response time of PMSE to ionospheric heating, J. Geophys. Res., 108, 8446, doi:10.1029/2002JD002385, 2003.

Biebricher, A., Havnes, O., Hartquist, T. W., and LaHoz, C.: On the influence of plasma absorption by dust on the PMSE overshoot effect, Adv. Space Res., 38(11), 2541-2550, doi:10.1016/j.asr.2005.02.061, 2006.

Birdsall C. K. and Langdon, A. B.: Plasma Physics via Computer Simulation, New York, McGraw-Hill, 1991.
Chen, C. and Scales, W. A.: Electron temperature enhancement effects on plasma irregularities associated with charged dust in the Earth's mesosphere, J. Geophys. Res., 110, A12313, doi:10.1029/2005JA011341, 2005.

Chen, C. and Scales, W. A.: Active perturbation of dust associated electron irregularities in the Earth's mesosphere: Discrete charging effects, IEEE Trans. Plasma Sci., 35, 731-735, doi:10.1109/TPS.2007.895228, 2007.

Chen, F. R.: Introduction to Plasma Physics and Controlled Fusion, New York, Plenum, 1984.

Chilson, P. B., Belova, E., Rietveld, M. T., Kirkwood, S., and Hoppe, U.: First artificially induced modulation of PMSE using the EISCAT heating facility, Geophys. Res. Lett., 27, 38013804, 2000.

Cho, J. Y. N. and Rottger, J.: An updated review of polar mesospheric summer echoes; Observation, theory, and their relationship to noctilucent clouds and subvisible aerosols, J. Geophys. Res., 102, 2001-2020, 1997.

D'Angelo, N.: Ion and Dust Acoustic Waves in Polar Mesospheric Summer Echoes, Phys. Lett. A., 336, 204-209, 2005.

Eklund, W. L. and Balsley, B. B.: Long-term observations of the Artic mesophere with MST radar at Poker Flat, Alaska, J. Geophys. Res. 8, 7775-7784, 1981.

Havnes, O., Troim, J., Blix, T., Mortensen, W., Naesheim, L. I., Thrane, E., and Tonnesen, T.: First detection of charged dust particles in the Earth's mesosphere, J. Geophys. Res., 101, 10839 $10847,1996$.

Havnes, O., La Hoz, C., and Naesheim, L. L.: First Observations of the PMSE overshoot effect and its use for investigating the conditions in the summer mesosphere, Geophys. Res. Lett., 30, 2229, doi:10.1029/2003GL018429, 2003.

Havnes, O.: Polar mesospheric Summer Echoes (PMSE) overshoot effect due to cycling of artificial electron heating, J. Geophys. Res., 109, A02309, doi:10.1029/2003JA010159, 2004.

Lie-Svenson O., Blix, T. A., and Hoppe, U.-P.: Modeling the plasma response to small-scale aerosol particle perturbations in the mesopause region, J. Geophys. Res., 108(D8), 8442, doi:10.1029/2002JD002753, 2003.

Rapp, M. and Lubken, J.-F.: On the nature of PMSE:, Electron diffusion in the vicinity of charged paricles revisited, J. Geophys. Res., 108, 2857-2870, 2003.

Scales, W.: Electron temperature effects on small-scale plasma irregularities associated with charged dust in the earth's mesosphere, IEEE Trans. Plasma Sci., 32, 724, doi:10.1109/TPS.2004826082, 2004.

Scales, W. A. and Chen, C.: On the initial perturbation of mesospheric dust associated by high power radio waves, Adv. Space Res., 41(1), 50-56, doi:10.1016/j.asr.2007.08.009, 2007. 